\title{
BMJ Open Acceptance and commitment therapy for young brain tumour survivors: study protocol for an acceptability and feasibility trial
}

\author{
Sam Malins (D) , ${ }^{1,2}$ Ray Owen, ${ }^{3}$ Ingram Wright, ${ }^{4,5}$ Heather Borrill, ${ }^{6}$ Jenny Limond, ${ }^{7}$ \\ Faith Gibson, ${ }^{8,9}$ Richard G Grundy, ${ }^{10}$ Simon Bailey, ${ }^{6,11}$ Steven C Clifford, ${ }^{6,11}$ \\ Stephen Lowis, ${ }^{4,5}$ James Lemon, ${ }^{12}$ Louise Hayes, ${ }^{13}$ Sophie Thomas ${ }^{1}$
}

To cite: Malins S, Owen R, Wright I, et al. Acceptance and commitment therapy for young brain tumour survivors: study protocol for an acceptability and feasibility trial. BMJ Open 2021;11:e051091. doi:10.1136/ bmjopen-2021-051091

- Prepublication history and additional supplemental material for this paper are available online. To view these files, please visit the journal online (http://dx.doi.org/10.1136/ bmjopen-2021-051091)

Received 09 March 2021 Accepted 24 May 2021
Check for updates

(C) Author(s) (or their employer(s)) 2021. Re-use permitted under CC BY-NC. No commercial re-use. See rights and permissions. Published by BMJ.

For numbered affiliations see end of article.

Correspondence to

Dr Sam Malins;

sam.malins@nottingham.ac.uk

\section{ABSTRACT}

Introduction Survivors of childhood brain tumours have the poorest health-related quality of life of all cancer survivors due to the multiple physical and psychological sequelae of brain tumours and their treatment. Remotely delivered acceptance and commitment therapy (ACT) may be a suitable and accessible psychological intervention to support young people who have survived brain tumours. This study aims to assess the feasibility and acceptability of remotely delivered ACT to improve quality of life among these young survivors.

Methods and analysis This study is a two-arm, parallel group, randomised controlled trial comparing ACT with waitlist control at 12-week follow-up as the primary endpoint. Seventy-two participants will be recruited, who are aged 11-24 and have completed brain tumour treatment. Participants will be randomised to receive 12 weeks of ACT either immediately or after a 12-week wait. The DNA-v model of ACT will be employed, which is a developmentally appropriate model for young people. Feasibility will be assessed using the proportion of those showing interest who consent to the trial and complete the intervention. Acceptability will be assessed using participant evaluations of the intervention, alongside qualitative interviews and treatment diaries analysed thematically. A range of clinical outcome measures will also assess physical and mental health, everyday functioning, quality of life and service usage at 12-week follow-up. The durability of treatment effects will be assessed by further follow-up assessments at 24 weeks, 36 weeks and 48 weeks.

Ethics and dissemination Ethical approval was given by East Midlands, Nottingham 1 Research Ethics Committee (Reference: 20/EM/0237). Study results will be disseminated in peer-reviewed journals, through public events and relevant third sector organisations. Trial registration ISRCTN10903290; NCT04722237.

\section{INTRODUCTION}

Due to treatment advances, childhood brain tumour survival rates continue to improve, but survival is often associated with significant morbidity. Physical symptoms such as fatigue, and cognitive deficits such as memory
Strengths and limitations of this study

- Key feasibility and acceptability outcomes are assessed in an identified research priority area, but the study will also provide initial evidence for the clinical and cost-effectiveness of acceptance and commitment therapy for young people who have experienced brain tumour treatment.

- The study employs a mixed methods design that aims to integrate standardised clinical assessments with qualitative analysis of patients' experiences of individual sessions (using diaries) and overall treatment (using interviews).

- As a feasibility and acceptability study, it cannot offer definitive evidence of clinical effectiveness.

difficulties are commonly coupled with psychological problems such as anxiety and post-traumatic stress. ${ }^{1}$ Consequently, people who survive childhood brain tumours are identified as having the poorest quality of life of all cancer survivors. ${ }^{2}$ In the context of the COVID-19 pandemic, disrupted education, social isolation, financial loss to parents/ carers and direct COVID-19-related ill-health also has an especially severe impact on young brain tumour survivors, due to pre-existing vulnerability. ${ }^{3}$

The identification of a psychological care package to improve well-being, social functioning and mental health is regarded as the top research priority by young people who have experienced cancer and their carers. ${ }^{4}$ Despite this, there are few studies of psychological therapies for brain tumour patients and none spanning the developmentally important transition from childhood to adulthood.

Acceptance and commitment therapy (ACT) is an evidence-based psychological therapy that has been used to improve 
physical and mental health among adults with long-term conditions, including cancer. ${ }^{5}$ It fosters engagement with, rather than avoidance of, painful experiences, to move towards acceptance of unchangeable difficulties alongside building a rich and meaningful life despite the presence of ongoing problems. Furthermore, the acceptance-type responses encouraged in ACT are associated with improved post-treatment functioning compared with other response styles, such as suppression, in adult patients with brain tumour. ${ }^{6}$ This gives ACT face validity for application to brain tumour survivors, where there can be permanent cognitive impairment and unavoidable, ongoing physical symptoms. DNA-v is an adaptation of ACT that is showing greater acceptability among young people. ${ }^{7}$ However, there has been no published ACT intervention research with young people surviving brain tumours to date.

Many young people who survive brain tumours receive care on a regional basis from principal treatment centres which are often a long journey from home, challenging equity of access to psychological care. Regular psychological therapy sessions at such centres cause additional disruption to patients' lives during the post-treatment phase, when they are typically aiming to re-engage with normal life. Remote delivery of psychological therapy can improve accessibility to treatment for those who would not otherwise seek psychological care and outcomeeffectiveness can be similar to face-to-face treatment. ${ }^{8-10}$ In addition, during the COVID-19 pandemic there have been calls for mental healthcare to be enhanced for vulnerable groups specifically including the use of remotely delivered mental health treatment. ${ }^{11}$

In summary, the psychosocial needs of people surviving childhood brain tumours are often not explicitly treated, resulting in poorer quality of life. A suitable modality for a psychological intervention is ACT, due to its focus on living well despite continuing difficulties. Given the ongoing COVID-19 restrictions and geographical organisation of brain tumour services, remote therapy is likely to be the most suitable and accessible delivery medium.

This study aims to assess the feasibility and acceptability of ACT as a psychological treatment to improve quality of life among adolescents and young adults after brain tumour treatment. Active intervention will be compared with a waitlist control group after 12 weeks follow-up. The durability of treatment effects will be assessed over a 12-month follow-up period. The intervention will be delivered via video conferencing and will therefore also assess the feasibility and acceptability of video conferencing delivery of ACT for this population. The secondary aim will be a preliminary assessment of clinical and costeffectiveness of ACT.

\section{METHOD}

\section{Design}

This study is a two-arm, parallel group, randomised controlled trial comparing ACT with waitlist controls who will then receive ACT after the 3-month waiting period. Participants will be randomised on a 1:1 ratio to receive 12 weeks of treatment either immediately or following a 12-week wait. The treatment will be DNA-v which is a model of ACT adapted to those aged 11-24 years. The model will be further adapted for those who have undergone brain tumour treatment. As trial therapists will perform assessments, blinding is not possible. Follow-up assessments will be conducted at 12 weeks, 24 weeks, 36 weeks and 48 weeks post-randomisation with primary endpoint at 12 weeks. The later follow-ups will be used to assess the durability of any clinical effects. No restrictions will be placed on access to other services during study participation, but service use will be recorded. Recruitment started 04 January 2021 and is planned to end 31 January 2023.

A subsample of participants will be invited to participate in an embedded process evaluation, which will include semi-structured interviews with 7-10 participants from each arm of the trial after their post-treatment follow-up. Purposive sampling will be used to gain representation across trial arms, age, gender, ethnicity, intervention engagement and intervention satisfaction. This qualitative data will be complemented by post-session reflective video or written diary data, in which participants will briefly reflect on each session and what they found most helpful (figures 1 and 2).

\section{Patient and public involvement}

Young people with lived experience of brain tumours have been involved in the study from the initial funding application and review. Since then, a group of nine young people with lived experience of brain tumours have contributed to a patient advisory group, facilitated by the chief investigator and trial manager and assisted by a member of the brain tumour charity patient support team. All key decisions in developing and running the trial involve separate discussion with the Patient Advisory Group prior to discussion with the trial management group (TMG; which also includes PAG membership). The PAG has opportunity to raise ideas, concerns or questions independent of the TMG agenda.

Specifically, patient feedback has been used to design the recruitment and assessment processes to ensure that there is sufficient opportunity for participants to choose the level of parental involvement, particularly for those aged 16-24. Given the ongoing inclusion of patient representatives in the research team, their advice will inform dissemination plans that will enable the greatest reach to relevant patient groups and services.

\section{Participants}

Using a reference study of ACT for adolescents, ${ }^{12}$ to detect a clinically important average difference of $0.5 \mathrm{SD}$ between intervention and waitlist control with $80 \%$ power at $\mathrm{p}<0.05$ (two-tailed), requires a sample size of 72 participants allowing $20 \%$ dropout. 


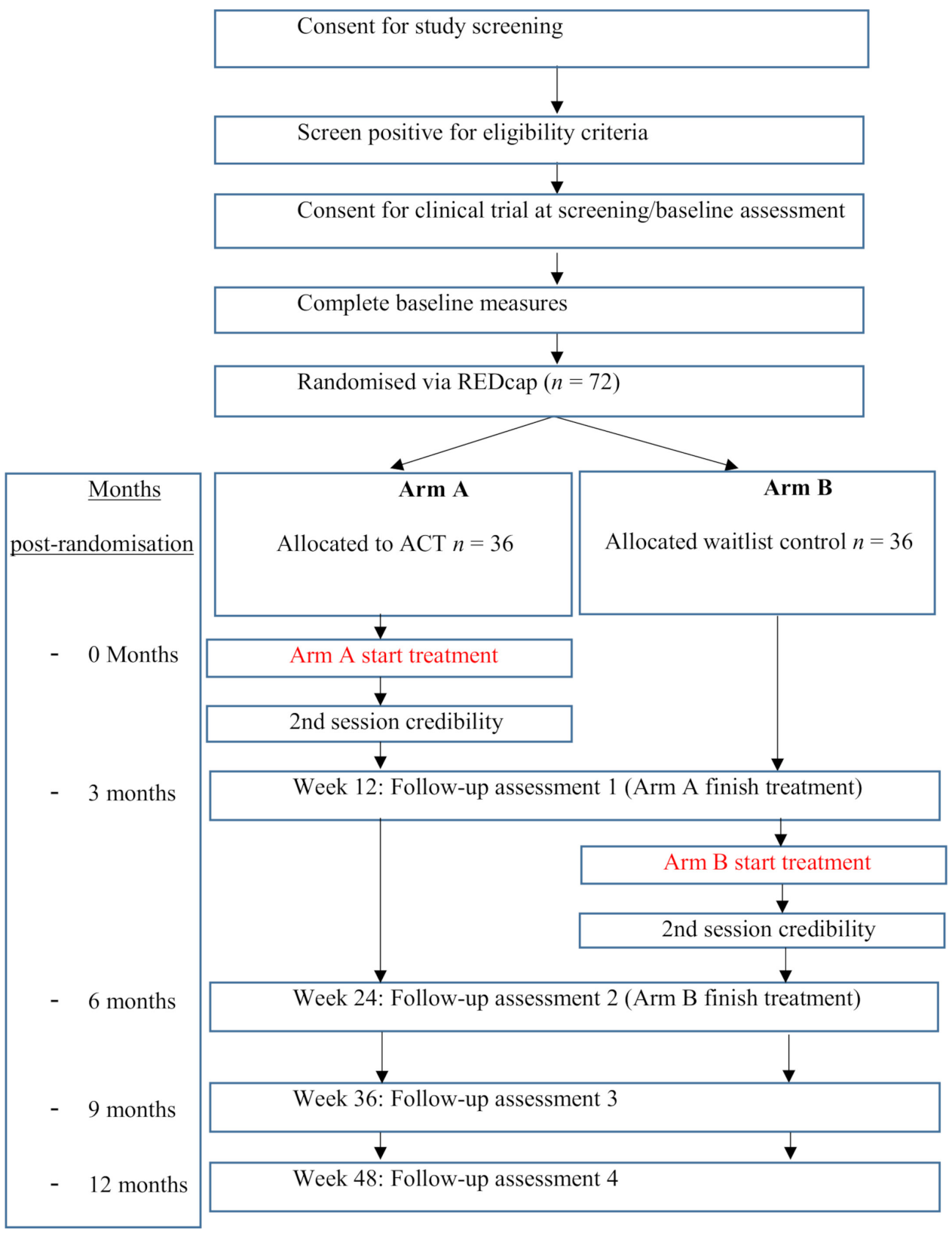

Figure 1 Trial flowchart. ACT, acceptance and commitment therapy; REDCap, Research Electronic Data Capture.

Inclusion criteria

Participants will be included if they meet the following criteria:

- Aged 11-24 years at the time of randomisation.

- Received treatment for a brain tumour at a participating principle treatment centre.

- Active brain tumour treatment is complete and their condition stable for at least 6 months

- Have sufficient cognitive ability to engage with ACT sessions as judged by the clinician at baseline assessment.

- Competent to provide informed consent (participants aged 16 or over) or assent (participants aged 11-15).

- Parent/carer competent to provide informed consent (for participants aged 11-15).
Exclusion criteria

Participants will be excluded if affected by the following criteria:

- Received a structured behavioural intervention within 6 months prior to study recruitment.

- Previous or current alcohol/substance dependence, psychosis, suicidality or eating disorder

- Moderate or severe intellectual disability, confirmed through researcher judgement at screening through questions relating to school type and previous diagnoses.

- Immediate risk to self or others.

- The patient or their parent/carer is not able to speak, read or write English. 


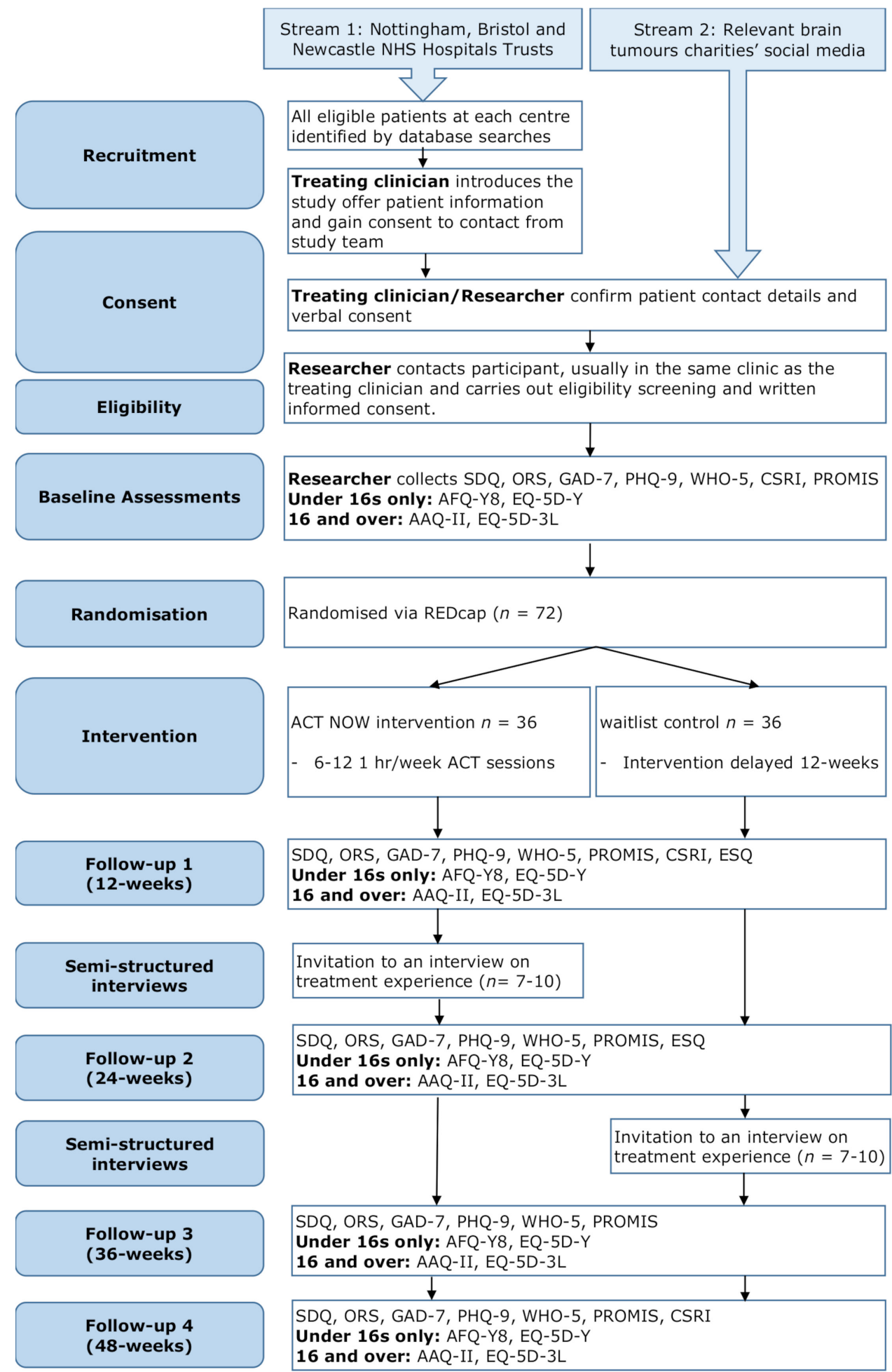

Figure 2 Trial procedures. AAQ-II, Acceptance and Action Questionnaire V.2; ACT, acceptance and commitment therapy; AFQ-Y8, Avoidance and Fusion Questionnaire for Youth; CSRI, Client Service Receipt Inventory; EQ-5D-Y, EuroQol 5-dimension youth version; EQ-5D-3L, EuroQol 5-dimensions 3-levels; ESQ, Experience of Service Questionnaire; GAD-7, Generalised Anxiety Disorder 7-items; ORS, Outcome Rating Scale; PHQ-9, Patient Health Questionnaire; PROMIS, Patient-Reported Outcomes Measurement Information System; REDCap, Research Electronic Data Capture; SDQ, Strengths and Difficulties Questionnaire; WHO-5, WHO Well-Being Index 5-items.

\section{Study setting and recruitment}

Participants will be recruited from one of three usual care oncology teams based at three principle treatment centres covering different regions of England. Oncology staff will approach eligible patients and seek consent for a researcher to contact them. Participants will also be recruited via public advertisements in hospital clinics and national cancer charity publicity. If consent to contact is 
given, or patients make contact via advertising, patients (and parents/carers for patients under 16) will be sent age-appropriate study information and a screening assessment will be arranged to assess eligibility. At the screening assessment informed consent will be sought either remotely or face-to-face prior to enrolment (see supplementary materials for model consent forms). Patients will then be randomised at enrolment using the REDCap (Research Electronic Data Capture; https:// www.redcapcloud.com/) secure cloud storage system. Researchers completing assessments will be qualified clinical psychologists.

\section{Assessments}

\section{Screening}

The screening questionnaire assesses patient eligibility. Where patients are aged over 16 they will be given the choice of whether they wish to involve their parent/ carer. For participants aged 11-15, the questionnaire will be completed jointly with their parent/carer initially and verified separately with the patient alone. The 5 min screening questionnaire asks about brain tumour history and treatment, contact with healthcare services, other diagnoses/problems, type of education facility attended, age of participant and other eligibility criteria.

Patients who do not meet screening criteria because they have recently received a behavioural therapy within the specified exclusion time frame ( 6 months), may be eligible for rescreening at a later date when these time exclusions have passed. In which case, researchers will seek consent to contact these patients at an agreed later point to reassess eligibility.

\section{Feasibility}

Feasibility will be assessed by documenting the proportion of patients showing interest who then consent to the trial and complete the intervention. Completion is defined as attending five or more ACT sessions. Fidelity to the ACT therapeutic model will be assessed monthly and then bimonthly using the ACT fidelity measure. ${ }^{13}$

\section{Acceptability}

Acceptability will be assessed using the session attendance rate. An adapted 6-item patient-reported version of the Credibility/Expectancy Questionnaire ${ }^{14}$ will also be given at baseline and after the second ACT session to assess treatment credibility. Participants' experience of the intervention will be assessed using the Experience of Service Questionnaire, ${ }^{15}$ with an additional item to assess video conferencing treatment satisfaction.

\section{Session-by-session routine outcome monitoring}

Routine outcome monitoring involves monitoring change in therapeutic outcome session-by-session and offering therapists feedback on algorithm-based predicted post-treatment changes using similar outcome trajectories of previous patients. ${ }^{16}$ The approach is evidenced as best practice within psychological therapies in general and specifically for children and adolescents, because it reduces therapy failure, increases the speed of improvements, and may enhance the magnitude of improvement. ${ }^{17-19}$

Each ACT session will begin by completing the Outcome Rating Scale ${ }^{20}$ : a brief, general assessment of self-reported well-being. Each session will end by completing the Session Rating Scale ${ }^{21}$ : a brief assessment of therapeutic alliance.

\section{Psychological flexibility}

Psychological flexibility is the main treatment target in ACT, which is the ability to remain in contact with current experiences even when unwanted thoughts, feelings or sensations are present, while selecting actions that support one's personal values. ${ }^{22}$ Psychological flexibility is associated with improved physical and mental health, quality of life and functioning. ${ }^{23}$

\section{Acceptance and Action Questionnaire $\|^{24}$}

The Acceptance and Action Questionnaire (AAQ-II) is a 7-item self-reported assessment of psychological inflexibility for participants aged 16 and over. (eg, 'emotions cause problems in my life').

\section{Avoidance and Fusion Questionnaire for Youth 8-items ${ }^{25}$}

The Avoidance and Fusion Questionnaire for Youth 8-items (AFQ-Y8) will be used as an 8-item self-reported measure of psychological inflexibility for those aged 11-15 or older participants with mild intellectual disabilities (eg, 'my thoughts and feelings mess up my life').

\section{Mental health and well-being \\ WHO Well-Being Index 5-items ${ }^{26}$}

The WHO Well-Being Index 5-items (WHO-5) is a 5-item self-reported assessment of well-being and mental health suitable for children and adults (eg, 'over the past week, I have felt calm and relaxed').

Generalised Anxiety Disorder assessment 7-items ${ }^{27}$

The Generalised Anxiety Disorder 7-items (GAD-7) is a 7-item self-reported measure of generalised anxiety symptoms based on diagnostic criteria (eg, worrying too much about different things over the previous 2 weeks).

\section{Patient Health Questionnaire 9-items ${ }^{28}$}

The Patient Health Questionnaire 9-items (PHQ-9) assesses depression symptoms on nine self-reported items based on diagnostic criteria for major depression (eg, feeling down depressed or hopeless over the previous 2 weeks). The PHQ-9 is validated for use among adolescents and adults. ${ }^{29}$

\section{General health and quality of life \\ EuroQol 5-dimensions 3-levels ${ }^{30}$}

The EuroQol 5-dimensions 3-levels (EQ-5D-3L) is a self-reported assessment of health-related quality of life for participants aged 16-24. Each dimension is rated at 3-levels: no problems, some problems and extreme problems (eg, 'I have no problems with self-care'). 


\section{EuroQol 5-dimensions youth-version ${ }^{31}$}

The EuroQol 5-dimensions youth-version (EQ-5D-Y) is based on the EQ-5D-3L but uses age-appropriate language for participants (11-15 years old) (eg, 'I have no problems washing or dressing myself').

\section{Visual Analogue Scale ${ }^{30}$}

The Visual Analogue Scale (VAS) is a self-reported assessment of general health suitable for all participants using a 0-100 VAS, with 0 defined as the worst health imaginable and 100 as the best health imaginable.

\section{Patient-Reported Outcomes Measurement Information System,} Satisfaction with Social Roles and Activities ${ }^{32}$

The Patient-Reported Outcomes Measurement Information System (PROMIS) assesses self-reported satisfaction with performing one's usual social roles and activities for all participants (eg, 'I am satisfied with my ability to participate in family activities').

\section{Functioning}

\section{Strengths and Difficulties Questionnaire 25-item ${ }^{33}$}

The Strengths and Difficulties Questionnaire (SDQ is a 25-item patient-completed measure of behavioural and emotional functioning (and parent/carer-completed where the patient is under 16).

\section{Health service usage}

\section{Client Service Receipt Inventory ${ }^{34}$}

The Client Service Receipt Inventory (CSRI) is a flexible research instrument developed to collect information on service receipt, service-related issues and income. This modified version also combines elements of the child and adolescent service use schedule, ${ }^{35}$ such as school attendance. The CSRI is completed with the parent/carer unless the participant is seeking treatment independently, in which case the participant completes the CSRI.

\section{Assessment schedule}

Routine outcome monitoring is completed at each ACT session and clinical outcomes are collected at baseline, 12-week, 24-week, 36-week and 48-week follow-up for the PHQ-9, GAD-7, WHO-5, AAQ-II/AFQ-Y8, EQ-5D and PROMIS. The CSRI and the SDQ will only be completed at 12-week and 48-week follow-up (see table 1 for the schedule of assessments). All anonymised outcome data will be collected and stored on REDCap. Data management procedures will be aligned with those of the sponsoring NHS Trust.

\section{Treatment procedures}

The DNA-v therapeutic model is an adaptation of ACT specifically for adolescents and young adults, which aims to be developmentally relevant for this stage of life. ${ }^{7}$ The approach involves exploration of different skills to address difficulties. Specifically, the approach teaches participants how to manage their behaviour by grouping them into four skills. First, the V, stands for vitality and value and is used to help guide choices toward living with meaning despite difficult experiences such as cancer. 'The Discoverer' is used to learn how one can interact with the world on a trial and error basis, investigating and discovering through building strengths and creating new behaviours. 'The Noticer' describes one's observing experiences and learning to respond with awareness rather than being reactive; exploring with senses, body and awareness. 'The Advisor' is the skill of learning how we use our inner voice to problem solve, make sense of situations and make decisions based on previous experiences and learning history. Participants are encouraged to move between these roles and investigate what it is like to approach situations, including times of difficulty, in different ways. The overall aim is to learn how to choose actions that support a meaningful, purposeful life.

Involvement of parents/carers will be incorporated within the treatment protocol, particularly for younger participants. The level of parent/carer involvement will be negotiated individually. The treatment is primarily focused on individual work with the participant and joint session time intends to feedback learning to parents/ carers with the aim of negotiating ways of integrating helpful aspects of therapy into home life.

Participants will be coached in the use of video conferencing software to maximise therapeutic benefits. This will include fluent use of screen-sharing and sharing computer software facilities. This aims to support therapists and participants to use all activities that might be included in a face-to-face consultation when working remotely. Education will also involve agreement on simple contingency management plans for managing video conferencing connection failure and agreed signals if confidentiality is compromised.

Using online software (https://www.myoutcomesukapp.com/) throughout therapy, brief measures of patient rated outcome and patient experience of each session will be used to evaluate and guide treatment progress. ${ }^{19}$ These processes ensure that therapeutic problems are identified and addressed quickly.

Personalised, targeted smart messaging may improve retention in psychological therapy and outcomes after the completion of therapy. ${ }^{36}{ }^{37}$ Therefore, relapse prevention plans will be organised into brief weekly reminders that can be sent as text messages after therapy has finished. The messages received will be tailored to participantreported well-being each week, with messages specific to times when they are doing well, experiencing early warning signs of relapse or experiencing full relapse.

\section{METHOD OF ANALYSIS}

\section{Primary outcome analysis}

All baseline variables will be summarised by randomised group. Categorical data will be reported as frequencies (\%) and continuous data will be reported as mean (SD); unless skewed then they will be reported as median (IQR). Any baseline characteristics that are seen to differ 
Table 1 Standard Protocol Items: Recommendations for Interventional Trials diagram of assessments at enrolment, allocation, weekly sessions and follow-up

\section{Study period}

\begin{tabular}{|c|c|c|c|c|c|c|c|c|c|}
\hline \multirow[b]{3}{*}{ Time points } & \multicolumn{9}{|c|}{ Study period } \\
\hline & \multirow[b]{2}{*}{ Enrolment } & \multirow[b]{2}{*}{ Baseline } & \multicolumn{7}{|c|}{ Post-allocation } \\
\hline & & & Session 1 & Session 2 & Session $\leq 12$ & $3 m$ & $6 m$ & $9 m$ & $12 \mathrm{~m}$ \\
\hline \multicolumn{10}{|l|}{ Enrolment: } \\
\hline Eligibility screening & $x$ & & & & & & & & \\
\hline Informed consent & $\mathrm{x}$ & & & & & & & & \\
\hline Allocation & & $x$ & & & & & & & \\
\hline \multicolumn{10}{|l|}{ Intervention: } \\
\hline \multicolumn{10}{|l|}{$\mathrm{ACT}$} \\
\hline \multicolumn{10}{|l|}{ Assessments: } \\
\hline Screening questionnaire & $x$ & & & & & & & & \\
\hline CEQ & & $\mathrm{x}$ & & $x$ & & & & & \\
\hline SDQ & & $x$ & & & & $x$ & & & $\mathrm{x}$ \\
\hline PHQ-9 & & $\mathrm{x}$ & & & & $x$ & $\mathrm{x}$ & $\mathrm{x}$ & $x$ \\
\hline GAD-7 & & $x$ & & & & $x$ & $x$ & $x$ & $\mathrm{x}$ \\
\hline WHO-5 & & $x$ & & & & $\mathrm{x}$ & $x$ & $\mathrm{x}$ & $x$ \\
\hline AAQ-II/AFQ-Y8 & & $x$ & & & & $x$ & $x$ & $x$ & $\mathrm{x}$ \\
\hline EQ-5D-Y/EQ-5D-3L & & $x$ & & & & $\mathrm{x}$ & $x$ & $x$ & $\mathrm{x}$ \\
\hline PROMIS & & $x$ & & & & $\mathrm{x}$ & $x$ & $\mathrm{x}$ & $\mathrm{x}$ \\
\hline ORS & & $x$ & $x$ & $x$ & $x$ & $x$ & $x$ & $x$ & $x$ \\
\hline SRS & & & $x$ & $x$ & $x$ & & & & \\
\hline CSRI & & $\mathrm{x}$ & & & & $x$ & & & $\mathrm{x}$ \\
\hline ESQ & & & & & & $x$ & $\mathrm{x}$ & & \\
\hline
\end{tabular}

AAQ-II, Acceptance and Action Questionnaire-II; ACT, acceptance and commitment therapy; AFQ-Y8, Avoidance and Fusion Questionnaire for Youth; CEQ, Credibility/Expectancy Questionnaire; CSRI, Client Service Receipt Inventory; EQ-5D-3L, EuroQol 5-dimensions 3-levels; EQ-5D-Y, EuroQol 5-dimension youth version; ESQ, Experience of Service Questionnaire; GAD-7, Generalised Anxiety Disorder 7-items; ORS, Outcome Rating Scale; PHQ-9, Patient Health Questionnaire 9-items; PROMIS, Patient-Reported Outcomes Measurement Information System - Satisfaction with Social Roles and Activities; SDQ, Strengths and Difficulties Questionnaire; SRS, Session Rating Scale; ; WHO-5, WHO Well-Being Index 5-item.

between the groups will be included in multivariate models to control for confounding by these variables.

Consent will be obtained to use the data of participants who discontinue up until the point they leave the trial. All analysis will be based on observed data, no missing data will be imputed.

The primary outcomes are summarised descriptively:

1. The percentage of patients eligible and approached for the trial who went on to receive at least one session of treatment.

2. The percentage of patients completing treatment (attending at least five treatment sessions).

3. Patient rated experience of the treatment, benchmarked against national database ratings of National Health Service (NHS) child and adolescent psychological therapy services.

\section{Secondary outcome analysis}

Assuming normally distributed and balanced data, independent t-tests will compare clinical outcomes between arms at 3-month follow-up as the primary endpoint. If there are imbalances between arms multiple linear regression will be used to control for baseline differences.

To assess whether post-treatment changes are maintained during the follow-up period one-way repeated measures analysis of variances will be completed separately for each arm across 9-month and 6-month posttreatment follow-up for the two arms.

If the baseline characteristics of participants in the two arms are imbalanced and there is significant clustering of covariance within participants across assessment time points multilevel modelling will be used to nest follow-up outcomes within reporting participants hierarchically. This accounts for both within and between participant change over time giving a more accurate estimate of change across follow-up. ${ }^{38}$ Where the participant sample may be split by age (eg, the AAQ-II and AFQ-Y8) logistic regression will be used to report the OR of achieving minimal clinically important 
improvement between treatment group and waitlist control at 3-month follow-up. ${ }^{39}$ Logistic regression analyses will also account for group-level imbalances at baseline.

\section{Qualitative methods}

Qualitative analysis will be used on purposively sampled interviews and reflective written or video diary entries made by participants directly after each ACT session. Participants will be invited to either remain on the video call after the therapist has left or type on the reflective diary template brief reflections on the session and what they took from it. This qualitative data will be analysed using thematic analysis. ${ }^{40} 41$ Thematic analysis has been selected as a flexible analytical method which allows for both inductive (emerging from the text) and deductive (guided by theory) coding. Interviews and video diary entries will be recorded and transcribed verbatim. Data will be analysed using NVivo to establish themes and subthemes. A theme template will be established and an independent researcher will be asked to code a selection of text extracts to establish the trustworthiness of themes. ${ }^{42}$ The theme template will be revised following the independent data audit and consensus discussion among researchers. A mixed methods approach will be used to integrate qualitative and qualitative data in order to explore full implementation of the intervention. This will include integration of the participants' evaluation of service questionnaire with interview transcript data, diary reports and session attendance rate. This triangulation aims to use the reciprocal effect of session attendance, evaluation of treatment and qualitative reports to gain a fuller understanding of processes facilitating or impeding the feasibility of the intervention.

\section{Economic evaluation}

The primary cost-effectiveness analysis will be the mean incremental cost per quality-adjusted life-year of waitlist control compared with active treatment at 12-week follow-up, from an NHS and personal social services cost perspective. This will be derived from the health utility index using the EQ-5D and service use costs using the CSRI.

To calculate the mean cost per patient of ACT we will collect detailed information on therapist time and evaluate the cost of remote delivery. To establish the cost of usual treatment and other healthcare costs in both the intervention and comparator arms additional health and social care resource use will be obtained from a modified version of the CSRI. Unit costs will be taken from national published sources.

For each resource used we will report descriptive statistics for the percentage number of patients that accessed the service and the mean and SD of times they accessed. As part of the secondary analyses we will include cost of education and out-of-pocket costs.
ETHICS AND DISSEMINATION

East Midlands, Nottingham 1 Research Ethics Committee gave ethical approval (Reference: 20/EM/0237).

\section{Trial monitoring and safety \\ TMG}

The TMG consists of the study co-investigators and at least two patient advisors with lived experience of brain tumours. The TMG will meet at least every 6 months to discuss study progress and the trial conduct.

\section{Trial steering committee}

The trial steering committee (TSC) is formed of a medical statistician, an oncologist, clinical psychologists and is chaired by a clinical academic neuropsychologist. The TSC will provide independent supervision for the trial on behalf of the trial sponsor and funder. Every 6 months the TSC will review: trial progress, adherence to the protocol, patient safety and the consideration of new information of relevance to the research question.

The trial funders and sponsor will not contribute to the collection of data, study management, analysis or interpretation of data, writing of reports or the decision to submit reports for publication (sponsor email address: researchsponsor@nuh.nhs.uk). Any changes to the study protocol would need to be agreed by the TMG, the TSC and the research ethics committee.

\section{Recording adverse events}

This study is deemed to be low risk in terms of adverse events related to treatment. There are no drug treatments involved and the therapy trialled is well-established as safe and effective. Nonetheless, serious adverse events (SAEs) that are unexpected and directly related to the intervention will be recorded in the medical records or other designated place following consent. These events will be recorded with clinical symptoms and accompanied with a description of the event. Safeguarding issues will be recorded and reported to the chief investigator immediately and appropriate local NHS safeguarding procedures will be followed.

\section{Reporting SAEs}

Participants will be asked to contact the study site immediately after any adverse events and will be asked if any have occurred at follow-up assessments. Any adverse events reported will be assessed to see if it meets the criteria for an SAE that is unexpected and directly related to the intervention. All treatment-related SAEs will be recorded and reported to the research ethics committee as part of the annual reports. Unexpected SAEs will be reported within stipulated time frames. Participants who experience an SAE that is unexpected and related to the intervention may be withdrawn from the study at the discretion of the investigator and sponsor. Every withdrawal will be examined by the TSC.

\section{Dissemination}

The dissemination strategy employs a number of routes with aim of maximising the reach to study stakeholders. 
Findings will be reported in peer-reviewed journals and at related academic and clinical conferences. Results will also be disseminated to third sector organisations that offer support to young people who have experienced brain tumours. An end of study dissemination event will be held, which will include patients, carers, researchers, clinicians, clinical service managers and commissioners to support ongoing development beyond feasibility, if appropriate, in ways that are most relevant to patents and health services.

\section{DISCUSSION}

This study will provide an initial assessment of acceptability and feasibility of remotely delivered ACT as a psychological treatment to support young people after brain tumour treatment, coupled with initial evidence of cost and clinical effectiveness. The study will also obtain rich data on patient experience of remote delivery of psychological therapy, which will offer important insights on patients' perspectives of new ways of working. Overall, this study will provide sufficient evidence to evaluate whether a larger-scale trial is warranted and will inform future trial design.

\section{Author affiliations}

${ }^{1}$ Nottingham University Hospitals NHS Trust, Nottingham, UK

${ }^{2}$ Division of Psychiatry and Applied Psychology, University of Nottingham,

Nottingham, UK

${ }^{3}$ Wye Valley NHS Trust, Hereford, UK

${ }^{4}$ University Hospitals Bristol and Weston NHS Foundation Trust, Bristol, UK

${ }^{5}$ Population Health Sciences, University of Bristol, Bristol, UK

${ }^{6}$ Newcastle upon Tyne Hospitals NHS Foundation Trust, Newcastle upon Tyne, UK

${ }^{7}$ College of Life and Environmental Sciences, University of Exeter, Exeter, UK

${ }^{8}$ Faculty of Health and Medical Sciences, University of Surrey, Guildford, UK

${ }^{9}$ Great Ormond Street Hospital For Children NHS Foundation Trust, London, UK

${ }^{10}$ Children's Brain Tumour Research Centre, University of Nottingham, Nottingham, UK

${ }^{11}$ Newcastle University, Newcastle upon Tyne, UK

${ }^{12} \mathrm{NHS}$ Dumfries and Galloway, Dumfries, UK

${ }^{13}$ DNA-v International, Melbourne, Victoria, Australia

Acknowledgements The authors would like to thank all members of the Brain Tumour Charity's Young Ambassadors who have contributed to the design and development of this study. The authors thank Dr Andrea Venn, University of Nottingham School of Medicine statistics service, for her support in designing the analysis.

Contributors ST, RO, IW, HB, JL, FG, RGG, SCC, SB and SL contributed to the conception, planning, design and grant application for the study. ST, SM, R0, LH and $\mathrm{JL}$ contributed to designing the intervention and related processes. All authors contributed drafting, revising and approving the manuscript.

Funding This study was supported by the Brain Tumour Charity (grant number GN-000645).

Competing interests None declared.

Patient consent for publication Not required.

Provenance and peer review Not commissioned; peer reviewed for ethical and funding approval prior to submission.

Supplemental material This content has been supplied by the author(s). It has not been vetted by BMJ Publishing Group Limited (BMJ) and may not have been peer-reviewed. Any opinions or recommendations discussed are solely those of the author(s) and are not endorsed by BMJ. BMJ disclaims all liability and responsibility arising from any reliance placed on the content. Where the content includes any translated material, BMJ does not warrant the accuracy and reliability of the translations (including but not limited to local regulations, clinical guidelines, terminology, drug names and drug dosages), and is not responsible for any error and/or omissions arising from translation and adaptation or otherwise.

Open access This is an open access article distributed in accordance with the Creative Commons Attribution Non Commercial (CC BY-NC 4.0) license, which permits others to distribute, remix, adapt, build upon this work non-commercially, and license their derivative works on different terms, provided the original work is properly cited, appropriate credit is given, any changes made indicated, and the use is non-commercial. See: http://creativecommons.org/licenses/by-nc/4.0/.

ORCID iD

Sam Malins http://orcid.org/0000-0001-9570-186X

\section{REFERENCES}

1 Turner CD, Rey-Casserly C, Liptak CC, et al. Late effects of therapy for pediatric brain tumor survivors. J Child Neurol 2009;24:1455-63.

2 Speechley KN, Barrera M, Shaw AK, et al. Health-Related quality of life among child and adolescent survivors of childhood cancer. J Clin Oncol 2006;24:2536-43.

3 Pfefferbaum B, North CS. Mental health and the Covid-19 pandemic N Engl J Med Overseas Ed 2020;383:510-2.

4 Aldiss S, Fern LA, Phillips RS, et al. Research priorities for young people with cancer: a UK priority setting partnership with the James Lind alliance. BMJ Open 2019;9:e028119.

5 Graham CD, Gouick J, Krahé C, et al. A systematic review of the use of acceptance and commitment therapy (act) in chronic disease and long-term conditions. Clin Psychol Rev 2016;46:46-58.

6 Aerts $\mathrm{H}$, Van Vrekhem T, Stas L, et al. The interplay between emotion regulation, emotional well-being, and cognitive functioning in brain tumor patients and their caregivers: an exploratory study. Psychooncology 2019;28:2068-75.

7 Hayes L, Ciarrochi J. The thriving adolescent. Oakland, CA: Context Press, 2015.

8 Carlbring P, Andersson G, Cuijpers P, et al. Internet-Based vs. face-to-face cognitive behavior therapy for psychiatric and somatic disorders: an updated systematic review and meta-analysis. Cogn Behav Ther 2018;47:1-18.

9 Thomas N, McDonald C, de Boer K, et al. Review of the current empirical literature on using videoconferencing to deliver individual psychotherapies to adults with mental health problems. Psychol Psychother 2021. doi:10.1111/papt.12332. [Epub ahead of print: 23 Feb 2021].

10 Fairburn CG, Patel V. The impact of digital technology on psychological treatments and their dissemination. Behav Res Ther 2017;88:19-25.

11 Rajkumar RP. COVID-19 and mental health: a review of the existing literature. Asian J Psychiatr 2020;52:102066.

12 Hayes L, Boyd CP, Sewell J. Acceptance and commitment therapy for the treatment of adolescent depression: a pilot study in a psychiatric outpatient setting. Mindfulness 2011;2:86-94.

13 O'Neill L, Latchford G, McCracken LM, et al. The development of the acceptance and commitment therapy fidelity measure (ACT-FM): a Delphi study and field test. J Contextual Behav Sci 2019;14:111-8.

14 Devilly GJ, Borkovec TD. Psychometric properties of the credibility/expectancy questionnaire. J Behav Ther Exp Psychiatry 2000;31:73-86.

15 Brown A, Ford T, Deighton J, et al. Satisfaction in child and adolescent mental health services: translating users' feedback into measurement. Adm Policy Ment Health 2014;41:434-46.

16 Wampold BE. Routine outcome monitoring: coming of age-With the usual developmental challenges. Psychotherapy 2015;52:458-62.

17 Lambert MJ, Whipple JL, Kleinstäuber M. Collecting and delivering progress feedback: a meta-analysis of routine outcome monitoring. Psychotherapy 2018;55:520-37.

18 Delgadillo J, de Jong K, Lucock M, et al. Feedback-informed treatment versus usual psychological treatment for depression and anxiety: a multisite, open-label, cluster randomised controlled trial. Lancet Psychiatry 2018;5:564-72.

19 Duncan BL, Reese RJ. The partners for change outcome management system (PCOMS) revisiting the client's frame of reference. Psychotherapy 2015;52:391-401.

20 Miller SD, Duncan B, Brown J. The outcome rating scale: a preliminary study of the reliability, validity, and feasibility of a brief visual analog measure. Journal of Brief Therapy 2003;2:91-100.

21 Duncan B, Miller S, Sparks JA. The Session Rating Scale: Preliminary psychometric properties of a "working" alliance measure. Journal of Brief Therapy 2003;3:3-12. 
22 Hayes SC, Strosahl K, Wilson KG. Acceptance and commitment therapy: the process and practice of mindful change. 2nd ed. London, UK: The Guilford Press, 2012.

23 Kashdan TB, Rottenberg J. Psychological flexibility as a fundamental aspect of health. Clin Psychol Rev 2010;30:865-78.

24 Bond FW, Hayes SC, Baer RA, et al. Preliminary psychometric properties of the acceptance and action Questionnaire-II: a revised measure of psychological inflexibility and experiential avoidance. Behav Ther 2011;42:676-88.

25 Greco LA, Lambert W, Baer RA. Psychological inflexibility in childhood and adolescence: development and evaluation of the avoidance and fusion questionnaire for youth. Psychol Assess 2008;20:93-102.

26 Allgaier A-K, Pietsch K, Frühe B, et al. Depression in pediatric care: is the WHO-Five well-being index a valid screening instrument for children and adolescents? Gen Hosp Psychiatry 2012;34:234-41.

27 Spitzer R, Kroenke K, Williams JMG. A brief measure for assessing generalised anxiety disorder; the GAD-7. Archives of Internal Medicine 2006;146:1092-7.

28 Kroenke K, Spitzer RL, Williams JB. The PHQ-9: validity of a brief depression severity measure. J Gen Intern Med 2001;16:606-13.

29 Richardson LP, McCauley E, Grossman DC, et al. Evaluation of the patient health Questionnaire-9 item for detecting major depression among adolescents. Pediatrics 2010;126:1117-23.

30 EuroQol Group. EuroQol--a new facility for the measurement of health-related quality of life. Health Policy 1990;16:199-208

31 Wille N, Badia X, Bonsel G, et al. Development of the EQ-5D-Y: a child-friendly version of the EQ-5D. Qual Life Res 2010;19:875-86.

32 Hahn EA, Beaumont JL, Pilkonis PA, et al. The PROMIS satisfaction with social participation measures demonstrated responsiveness in diverse clinical populations. J Clin Epidemiol 2016;73:135-41.
33 Goodman R. Psychometric properties of the strengths and difficulties questionnaire. J Am Acad Child Adolesc Psychiatry 2001;40:1337-45.

34 Beecham J, Knapp M. Costing Psychiatric Interventions. In: Thornicroft G, Brewin C, Wing J, eds. Measuring mental health needs. London: Gaskell, 2001: 200-24.

35 Byford S, Barrett B, Roberts C, et al. Cost-Effectiveness of selective serotonin reuptake inhibitors and routine specialist care with and without cognitive behavioural therapy in adolescents with major depression. Br J Psychiatry 2007;191:521-7.

36 Malins S, Biswas S, Patel S, et al. Preventing relapse with personalized smart-messaging after cognitive behavioural therapy: a proof-of-concept evaluation. Br J Clin Psychol 2020;59:241-59.

37 Wells C, Malins S, Clarke S, et al. Using smart-messaging to enhance mindfulness-based cognitive therapy for cancer patients: a mixed methods proof of concept evaluation. Psychooncology 2020;29:212-9.

38 Raudenbush SW, Bryk AS. Hierarchical linear models: applications and data analysis methods. 2nd ed. Thousand Oaks, CA: Sage Publications, 2002.

39 Jacobson NS, Truax P. Clinical significance: a statistical approach to defining meaningful change in psychotherapy research. $J$ Consult Clin Psychol 1991;59:12-19.

40 Braun V, Clarke V. Using thematic analysis in psychology. Qual Res Psychol 2006;3:77-101.

41 Braun V, Clarke V, Hayfield N. Thematic analysis. In: Cooper $\mathrm{H}$, Camic PM, Long DL, eds. Handbook of research methods in psychology, vol 2 research designs: quantitative, qualitative, neuropsychological, and biological. Washington, DC: American Psychological Association, 2012: 843-60.

42 Elliott R, Fischer CT, Rennie DL. Evolving guidelines for publication of qualitative research studies in psychology and related fields. $\mathrm{Br} J$ Clin Psychol 1999;38:215-29. 